\title{
Prenatal lipopolysaccharide increases maternal behavior, decreases maternal odor preference, and induces lipopolysaccharide hyporesponsiveness
}

\author{
Sandra Penteado ${ }^{1}$, Cristina de Oliveira Massoco-Salles Gomes ${ }^{3}$, Thiago Kirsten ${ }^{3}$, Thiago \\ Reis-Silva ${ }^{3}$, Rafael César de Melo ${ }^{1}$, Michelli Acenjo ${ }^{1}$, Nicolle Queiroz-Hazarbassanov ${ }^{3}$, and \\ Maria Martha Bernardi ${ }^{1,2}$ \\ 1- Universidade Paulista, São Paulo, SP, Brazil \\ 2- Universidade Federal do ABC, Santo André, SP, Brazil \\ 3- Universidade de São Paulo, São Paulo, SP, Brazil
}

\begin{abstract}
The present study investigated whether late maternal inflammation disrupts the mother/pup interaction, resulting in longlasting effects on pup behavior and alterations in biological pathways, thereby programming prepubertal behavior and the pups' inflammatory responses after bacterial endotoxin treatment. Female rats received $100 \mu \mathrm{g} / \mathrm{kg}$ lipopolysaccharide (LPS) or $.9 \%$ saline solution on gestation day 18. Reproductive performance was observed at birth. On lactation days (LD) 5 and LD 6, respectively, maternal behavior and maternal aggressive behavior were assessed. In pups, maternal odor preference on LD 7, open field behavior on LD 21, and serum tumor necrosis factor $\alpha$ (TNF- $\alpha$ ) levels after LPS challenge on LD 21 were investigated. The results showed that prenatal LPS exposure improved maternal care and reduced maternal aggressive behavior but did not alter maternal reproductive performance. Male offspring exhibited increased body weights at birth and reduced maternal odor preference. Lipopolysaccharide challenge increased the duration of immobility in the open field and induced a slight increase in serum TNF- $\alpha$ levels. Prenatal exposure to LPS during late pregnancy improved maternal care, reduced maternal olfactory preference, and induced TNF- $\alpha$ hyporesponsiveness to a single dose of LPS in pups. Keywords: prenatal inflammation, lipopolysaccharide, maternal behavior, maternal olfactory perception, tumor necrosis factor $\alpha$.
\end{abstract}

Received 25 August 2012; received in revised form 27 February 2013; accepted 1 March 2013. Available online 27 June 2013.

\section{Introduction}

Intrauterine infection and inflammation are known risk factors for brain injuries in neonates. Intrauterine inflammation leads to a dysregulation of the developing brain, known as fetal inflammatory response syndrome

Sandra H. N. W. Penteado, Rafael C. de Melo, Michelli K. Acenjo, and Maria Martha Bernardi, Programa de PósGraduação em Patologia Ambiental e Experimental e Programa de Pós-Graduação em Odontologia, Universidade Paulista, UNIP, Rua Dr. Bacelar 1212, CEP-04026-002, São Paulo, SP, Brazil. Maria Martha Bernardi, Centro de Matemática, Computação e Cognição, Universidade Federal do ABC, Rua Santa Adélia, 166, Torre 2, Bloco A, Sala 508-2, Santo André, SP, 09210-580, Brazil. Cristina de O. Massoco-Salles Gomes, Thiago B. Kirsten, Thiago M. Reis-Silva, and Nicolle QueirozHazarbassanov, Departamento de Patologia, Faculdade de Medicina Veterinária, Universidade de São Paulo, Av. Prof. Dr. Orlando Marques de Paiva, 87, São Paulo, SP, 05508-270, Brazil. Correspondence regarding this article should be directed to Maria Martha Bernardi, Centro de Matemática e Cognição, Universidade Federal do ABC, Rua Santa Adélia, 166, Bloco A, $5^{\circ}$ andar, Santo André, SP, CEP 09210-170, Brazil. Phone/Fax: +55 11 4996-7950. E-mail: marthabernardi@gmail.com (de Moura, Lisboa, \& Passos, 2008), regardless of the gestational age (Burd, Balakrishnan, \& Kannan, 2012). The maternal response to lipopolysaccharide (LPS) leads to a fetal inflammatory response mediated by cytokines that has been implicated in the development of a spectrum of neurodevelopmental disorders such as autism and schizophrenia (Meyer, 2011; Meyer et al., 2006).

Lipopolysaccharide is an endotoxin that originates from the cell wall of gram-negative bacteria, mimics bacterial infections, and is a potent inducer of inflammation (Avitsur, Pollak, \& Yirmiya, 1997; SalukJuszczak \& Wachowicz, 2005). Prenatal injections of LPS impair short- and long-term behavior and central nervous system activity in neonates (Boksa, 2010; Golan, Lev, Hallak, Sorokin, \& Huleihel, 2005; Meyer, Feldon, \& Fatemi, 2009). Previous studies from our group showed that prenatal LPS treatment $(100 \mu \mathrm{g} /$ $\mathrm{kg}$, intraperitoneally, on gestational day [GD] 9.5) in male offspring reduced social behavior in infancy and adulthood, decreased dopamine and metabolite levels in the striatum and decreased general activity in the 
open field after an LPS challenge without signs of permanent neuroinflammation (Kirsten et al., 2011; Kirsten, Taricano, Florio, Palermo-Neto, \& Bernardi, 2010b; Kirsten, Taricano, Maiorka, Palermo-Neto, \& Bernardi, 2010a). Interestingly, our research also showed that maternal behavior slightly improved in pregnant rats treated with LPS on GD 9.5 (Kirsten et al., 2010a), whereas treatment on GD 21 caused a reduction of maternal behavior (Bernardi et al., 2010). Additionally, prenatal LPS exposure on GD 14 to GD 20 decreased adult neurogenesis in the dentate gyrus, caused persistent microglial activation, downregulated transforming growth factor $\beta_{1}$ in the hippocampus, and impaired performance in the novel object recognition test (Graciarena, Depino, \& Pitossi, 2010).

Our hypothesis is that late maternal inflammation may disrupt the programming of prepubertal behavior and immune responses after additional inflammatory stimulation. Maternal care was also examined because alterations in maternal behavior may contribute to the long-term effects of stress on pup behavior (Carlos, Lemonica, de Grava Kempinas, \& Marques Pereira, 1996; Darnaudery \& Maccari, 2008).

Thus, $100 \mu \mathrm{g} / \mathrm{kg}$ LPS was administered to dams on GD 18. Maternal behavior was observed on lactation day (LD) 5, and maternal aggressive behavior was observed on LD 6. Maternal odor preference, open field behavior, and serum levels of the proinflammatory cytokine tumor necrosis factor $\alpha$ (TNF- $\alpha$ ) were observed in pups of the F1 generation after LPS challenge.

Maternal care was evaluated on LD 5 and LD 6 of the F1 generation because the degree of maternal behavior at these time points gradually decreases, and changes in maternal care can be revealed (Teodorov, Felício, \& Bernardi, 2010). Maternal odor preference reflects activity of a sensory system that is critical for mother/pup interactions (Slotnick \& Restrepo, 2005). We previously found that prenatal LPS treatment on GD 9.5 impaired maternal odor preference and cat odor aversion, both of which were related to decreased dopamine levels in the olfactory bulb (Kirsten et al., 2011). Finally, we administered $50 \mu \mathrm{g} /$ kg LPS intraperitoneally on LD 21 to challenge F1generation pups' immune systems and assess whether prenatal exposure to LPA alters behavior and TNF- $\alpha$ levels.

\section{Methods}

\section{Subjects}

Thirty-two pregnant Wistar rats (parental generation) between 12 and 13 weeks of age and weighing 230-255 g were used. Gestational day 0 was defined as the day when spermatozoa were detected in the vaginal smear. The dams were individually housed in polypropylene cages $(38 \times 32 \times 16 \mathrm{~cm})$ at a controlled temperature $\left(22 \pm 2^{\circ} \mathrm{C}\right)$ and humidity (65-70\%) with artificial lighting $(12 \mathrm{~h} / 12 \mathrm{~h}$ light/dark cycle; lights on at 6:00 AM). The animals had free access to Nuvilab rodent chow (Nuvital, Sao Paulo, SP, Brazil) and filtered water. Sterilized and residue-free wood shavings were used for the animal bedding. Two experiments were performed. In the first experiment, maternal performance, maternal aggressive behavior, and the pups' maternal odor preference were assessed. In this experiment, dams were divided into control (i.e., saline-treated) and experimental (i.e., LPS-treated) groups ( $n=8 \mathrm{dams} /$ group). The dams were allowed to give birth and nurture their offspring normally. The day of birth was designated postnatal day (PND) 1. No handling was performed on PND 1 to avoid maternal cannibalism. On PND 2, after weighing the litters and recording the number of pups, the litters were randomly culled to eight offspring (four males and four females; gender was determined by anogenital differences). No cross-fostering procedure was used. In the second experiment, eight dams per group were treated as in Experiment 1. On PND 21, littermates were separated and co-housed by sex under the same conditions as their parents. On this day, two males from each litter (F1 generation) received either $50 \mu \mathrm{g} / \mathrm{kg}$ LPS or saline solution, and open field behavior and serum TNF- $\alpha$ levels were assessed. Testing of the control and LPStreated rats was intermixed. The experimental design is summarized in Figure 1. Rats used in this study were maintained in accordance with the guidelines of the Committee on Care and Use of Laboratory Animal Resources, Paulista University, Brazil (protocol no. 014/09, CEUA-UNIP). These guidelines are similar to those of the U.S. National Institutes of Health. Experiments were performed in accordance with good laboratory practice protocols and quality assurance methods.

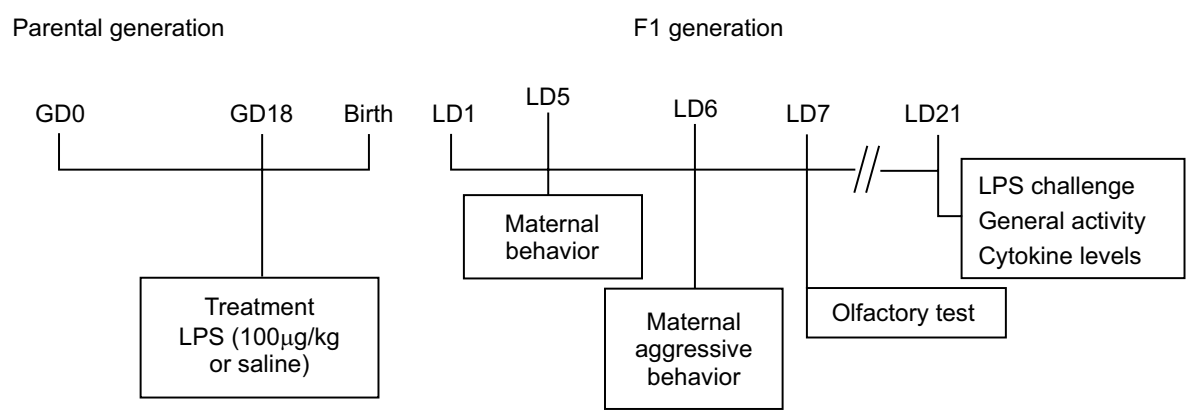

Figure 1. Experimental design. 


\section{Treatment}

Lipopolysaccharide from Escherichia coli (Sigma, St. Louis, MO, USA; serotype 0127:B8) was dissolved in sterile saline $(50 \mu \mathrm{g} / \mathrm{ml}$ LPS in $.9 \% \mathrm{NaCl}$ solution) and administered intraperitoneally to pregnant dams at a dose of $100 \mu \mathrm{g} / \mathrm{kg}$ on GD 18 ( $n=16 /$ group). This dose was chosen because it has been shown to (1) elicit sickness behavior, (2) induce endocrine alterations in dams, (3) increase cytokines at the placental level, (4) impair offspring birth rate, and (5) reduce social behavior in male offspring during infancy and adulthood (Kirsten, Taricano, Maiorka, Palermo-Neto, \& Bernardi, 2010b; Spencer, Mouihate, Galic, Ellis, \& 2007; Wang, Rousset, Hagberg, \& Mallard, 2006). The control group consisted of pregnant rats that received only sterile saline $(.9 \% \mathrm{NaCl})$ and underwent the same treatment schedule as LPS animals. Each control dam was treated with $.1 \mathrm{ml} / 100 \mathrm{~g}$ saline solution.

\section{Maternal studies}

Maternal reproductive performance. Maternal reproductive performance was observed on LD 2 in F1-generation dams that received LPS during gestation ( $n=8 \mathrm{dams} /$ group). The following parameters were assessed: number of pregnant females, total number of pups, number of male and female pups, number of pup deaths per litter, and litter weight.

Maternal behavior. Maternal behavior was analyzed as previously described (Bernardi, et al., 2010). Briefly, on LD 5 of the F1 generation ( $n=8$ dams/group), between 8:00 AM and 11:00 AM maternal behavior was observed in parental-generation female rats exposed to LPS on GD 18. These dams were used previously in the maternal retrieval test. Pups were removed from their dams, placed in another cage, and kept warm. Immediately following separation, the presence of a nest in the home cage was evaluated. Sixty minutes after maternal separation, all of the pups were returned to their mothers' cages, and maternal behavior examination began. The time to retrieve the first pup (in seconds), time to retrieve all of the pups (in seconds), time to group the pups (in seconds), duration of full maternal behavior (in seconds), and latency to exhibit maternal behavior were recorded. The dams were considered to display full maternal behavior if they transferred all of the pups to the nest and displayed nursing behavior with their back arched over the pups for three consecutive minutes. If the animals did not display full maternal behavior following $30 \mathrm{~min}$ of continuous observation, they were then checked every $15 \mathrm{~min}$ for $60 \mathrm{~min}$ and then hourly thereafter until full maternal behavior was observed.

Maternal aggressive behavior. The same dams that were observed for maternal behavior were used in this test. On LD 6 of the $\mathrm{F} 1$ generation ( $n=8 \mathrm{dams} /$ group), between 8:00 $\mathrm{AM}$ and 11:00 AM, maternal aggressive behavior was assessed in female rats exposed to LPS on GD 18. These rats were subjected to a 10-min maternal defense test (Wilkins, Logan, \& Kehoe, 1997). A male Wistar rat (i.e., intruder) was introduced into the home cage of the dam and offspring. Intruder rats were only used once. Behaviors against the intruder during the maternal defense test were recorded with a remote digital camcorder, and offensive behavior by the resident was later analyzed including the latency to the first attack (in seconds), attack frequency, total duration of attacks (in seconds), boxing frequency, and duration of boxing (in seconds). Maternal behavior in the presence of the intruder was also analyzed, including the frequency of carrying and hiding the pups and frequency of the intruder sniffing the pups.

\section{Pup studies}

Maternal odor preference test. Maternal odor preference was assessed in male pups on PND 7 as described elsewhere (Kirsten et al., 2011). Briefly, one male pup from each experimental and control litter $(n$ $=8$ per group) was examined. The experimental design was based on studies of associative olfactory learning and consisted of a two-odor choice between areas with nest material and fresh bedding. A polypropylene cage (38 $\times 32 \times 16 \mathrm{~cm}$ ) divided in half by a 2 -cm-wide neutral zone that ran the length of the box was used. In each area, $300 \mathrm{ml}$ of fresh or nest bedding was placed in adjacent corners. The pup was placed in the $2-\mathrm{cm}$ neutral zone at the end of the box, facing the opposite target bedding. During the 1-min trial, the amount of time that the pup spent with its head or whole body over each of the two areas was recorded. The number of pups that reached the area with and without odor was also assessed in each trial. A time of $60 \mathrm{~s}$ was recorded when the pups did not reach one of the sides. The animals were tested in five trials between 2:00 and 4:00 PM, with an intertrial interval of 2 min during which the pup was placed in its home cage. In each trial, the beddings were switched between the sides of the box. Following each test period, the box was cleaned with $5 \%$ ethanol to remove trace odors. The experiments were recorded with a video camera for later analysis. The pups' total time in each area was calculated by combining the times from the five trials.

General activity in the open field after LPS challenge. The general activity test was performed in F1-generation male pups that were prenatally exposed to LPS or saline solution on LD 21 ( $n=8$ pups/group) as described previously (Broadhurst, 1960). A challenge dose of LPS $(50 \mu \mathrm{g} / \mathrm{kg}$, i.p.) was administered 90 min before the experiments. This dose and time were chosen because they were reported to cause sickness behavior and the release of proinflammatory cytokines and glucocorticoids (Corrreia, Fernandes, \& Bernardi, 2008). Thus, the following groups were formed: prenatal saline and postnatal saline group ( $\mathrm{S}+\mathrm{S}$ group), prenatal saline and postnatal LPS group (S+LPS group), prenatal LPS and postnatal saline group (LPS+S group), and prenatal LPS and postnatal LPS group (LPS+LPS group). The open-field apparatus has been described in detail elsewhere (Broadhurst, 1960). Briefly, it consisted 
of a round wooden arena $(40 \mathrm{~cm}$ diameter, $40 \mathrm{~cm}$ high walls) that was painted black and divided into 25 parts. The apparatus was elevated $100 \mathrm{~cm}$ above the floor. For the observations, each rat was individually placed in the center of the apparatus between 2:00 and 4:00 PM. The following parameters were recorded: locomotor frequency (i.e., number of floor units entered), rearing frequency (i.e., number of times the animal stood on its hind legs), and immobility time (i.e., total time in seconds of lack of movement). The apparatus was washed with a $5 \%$ alcohol/water solution before the animals were placed into it to negate possible biasing effects caused by odor cues left by previous rats.

\section{Assessment of TNF $\alpha$ levels}

TNF- $\alpha$ levels were determined using the enzymelinked immunosorbent assay (ELISA). Sera of pups that were observed in the open field and challenged with LPS or saline were used in this experiment. A 96-well highbinding plate (Costar, Corning, NY, USA) was coated with mouse anti-rat TNF- $\alpha$ antibodies (R\&D Systems, Minneapolis, MN, USA); $4.0 \mu \mathrm{g} / \mathrm{ml}$ in phosphatebuffered saline [PBS]) at room temperature overnight. The plate was then washed three times with PBS that contained .05\% Tween-20 (Synth, Diadema, SP, Brazil) after each step. Nonspecific binding was blocked with PBS that contained $1 \%$ bovine serum albumin (BSA; Sigma-Aldrich, São Paulo, SP, Brazil). Serum samples $(100 \mu \mathrm{l})$ or standards $(0-4000 \mathrm{pg} / \mathrm{ml}$ recombinant rat TNF- $\alpha$; R\&D Systems) that were diluted in PBS with $1 \%$ BSA were incubated for $2 \mathrm{~h}$ at room temperature. Immunodetection was performed using biotinylated goat anti-rat TNF $\alpha$ antibodies $(225 \mathrm{ng} / \mathrm{ml}$ in PBS/1\% BSA; R\&D systems) for $2 \mathrm{~h}$ at room temperature followed by incubation with streptavidin-horseradish peroxidase for 20 min (1:200; R\&D Systems). The substrate solution was then applied for 15 min (OptEIA, BD, San Jose, CA, USA). The plate was read at $450 \mathrm{~nm}$, and the values were calculated in picograms per milliliter.

\section{Statistical analysis}

The dams were the experimental unit, and one or two males from each litter were used for each experiment with different animals in each experiment. The results are expressed as mean \pm SEM. Homoscedasticity was verified using an $F$-test or Bartlett's test. Normality was verified using the Kolmogorov-Smirnov test. Student's $t$-test (unpaired, two-tailed) was used to compare the parametric group data (i.e., maternal reproductive performance, pup body weight, maternal behavior, and maternal aggressive behavior). For percentage data, the $\chi^{2}$ test was used. A two-way analysis of variance followed by the Bonferroni post hoc test was used to analyze the time to reach one of the sides in the maternal odor preference test, the open field test data, and TNF- $\alpha$ levels. In all cases, values of $p<.05$ were considered statistically significant. Statistical analyses were performed using GraphPad Prism software, v.5 (GraphPad, San Diego, CA, USA).

\section{Results}

No alterations in reproductive parameters were found in females that were or were not prenatally treated with LPS (data not shown). Compared with the control group, the litter weight measured on LD 2 before litter standardization increased in the experimental group (control group: $49.58 \pm 1.16 \mathrm{~g}$; experimental group: $53.45 \pm 1.30 \mathrm{~g} ; p=.03$; mean $\pm \mathrm{SEM}$ ). No differences were observed between the number of pups born in each litter (control group: $9.18 \pm .66$; experimental group: $7.83 \pm 1.16 ; p=.29$; mean \pm SEM).

With regard to maternal behavior, LPS-treated female rats retrieved the first pup faster than females in the control group $(p=.044)$. The remaining parameters were not different between the control and experimental groups (Table 1). With regard to maternal aggressive behavior, female rats prenatally treated with LPS exhibited a decreased number of attacks $(p=.004)$ and total time of attacks $(p<.0001)$ compared with the control group. The remaining parameters were not different between the control and experimental groups (Table 1).

As depicted in Figure 2A, prenatal treatment with LPS reduced the number of pups that reached the side with maternal odor, without odor or remained in the neutral zone. The ANOVA revealed significant differences between treatments $\left(F_{1,42}=8.20, p=\right.$ $.0065)$ and side chosen $\left(F_{2,42}=39.18, p<.0001\right)$, with a significant interaction between factors $\left(F_{2,42}=24.6\right.$, $p<.0001)$. Compared with the control group, the experimental group exhibited a decreased number of pups that reached the odor area and the largest number of pups that remained in the neutral zone. Thus, pups prenatally exposed to LPS had impaired preference for the maternal odor. The time to reach the odor side in five trials decreased in the experimental pups compared with controls $(p<.029$; Figure 2B). The two-way ANOVA revealed that pups in the LPS group exhibited an increased amount of time to reach one of the sides only in the first session compared with the control group (treatment: $F_{1,70}=13.98, p=.0004$; sessions: $F_{4,70}=.77$, $p=.54$; interaction: $F_{4,70}=1.98, p=.11$; Bonferroni test, session 1: $p<.05$; Figure 2C). Thus, only in session 1 did the pups in the LPS group exhibit a decrease in locomotor function.

Figure 3 shows general activity in the open field in pups prenatally treated or not with LPS and challenged or not with $50 \mu \mathrm{g} / \mathrm{kg}$ LPS on LD 21. The two-way ANOVA revealed that locomotor frequency (postnatal treatment: $F_{1,28}=.02, p=.896$; prenatal treatment: $F_{1 / 28}$ $=.12, p=.734$; interaction: $F_{1,28}=.74, p=.395$; Figure $3 \mathrm{~A}$ ) and rearing frequency (postnatal treatment: $F_{1,28}=$ $.45, p=.501$; prenatal treatment: $F_{1,28}=.08, p=.773$; interaction: $F_{1,28}=1.29, p=.256$; Figure 3B) were not modified by the prenatal or postnatal treatments. The immobility duration (Figure 3C) was altered by postnatal treatment $\left(F_{1,28}=7.28, p=.02\right)$, but prenatal treatment did not affect immobility $\left(F_{1,28}=.23, p=.63\right)$. No interaction was observed between factors $\left(F_{1,28}=.14\right.$, 
Table 1. Maternal care of dams prenatally exposed to LPS $(100 \mu \mathrm{g} / \mathrm{kg}$, i.p)

\begin{tabular}{|c|c|c|c|}
\hline Parameter & Control group & LPS group & $P$ \\
\hline \multicolumn{4}{|l|}{ Maternal behavior } \\
\hline Retrieval $1^{s t}$ pup $(s)$ & $40.38 \pm 11.60$ & $13.25 \pm 3.89$ & 0.044 \\
\hline Retrieval all pups (s) & $202.63 \pm 43.26$ & $176.75 \pm 38.43$ & 0.662 \\
\hline Maternal position (min) & $21.26 \pm 3.93$ & $19.55 \pm 1.37$ & 0.687 \\
\hline Maternal position (\%) & 87.5 & 100 & 1.0 \\
\hline Grouping pups (\%) & 87.5 & 100 & 1.0 \\
\hline Presence of the nest (\%) & 100 & 100 & - \\
\hline \multicolumn{4}{|l|}{ Maternal aggressive behavior } \\
\hline First attack (s) & $147.73 \pm 24.00$ & $166.60 \pm 25.00$ & 0.595 \\
\hline Number of attacks & $6.65 \pm 0.70$ & $3.50 \pm 0.60$ & 0.004 \\
\hline Total number of attacks & $4.00 \pm 1.26$ & $3.00 \pm 1.40$ & 0.604 \\
\hline Time of fight (s) & $1.80 \pm 0.02$ & $1.00 \pm 0.05$ & $<0.0001$ \\
\hline Number of bits & $0.36 \pm 0.20$ & $0.40 \pm 0.24$ & 0.074 \\
\hline Number of times of retrieval pups & $0.27 \pm 1.00$ & $3.80 \pm 1.80$ & 0.109 \\
\hline Number of times hidden the pups & $1.54 \pm 0.66$ & $3.80 \pm 2.60$ & 0.414 \\
\hline Number of times the intruder sniffed the pups & $0.82 \pm 0.42$ & $0.40 \pm 0.24$ & 0.399 \\
\hline
\end{tabular}

Student $t$ test.

Note: Maternal behavior and maternal aggressive behavior were observed, respectively, on postnatal (PND) 5 and PND $6 . \mathrm{N}=8 /$ group.

Data are presented as mean $\pm \mathrm{SEM}$ or percentage

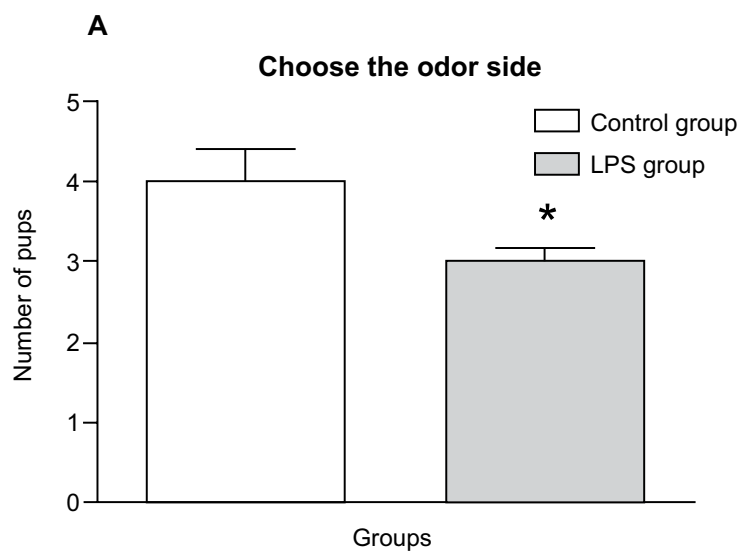

B

Time to reach the odor side

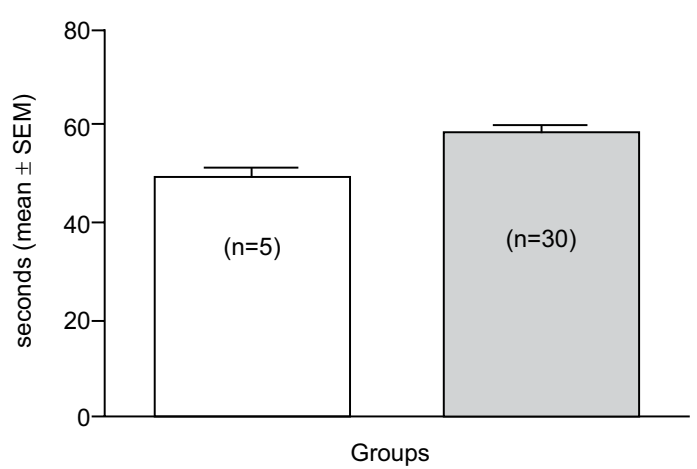

C

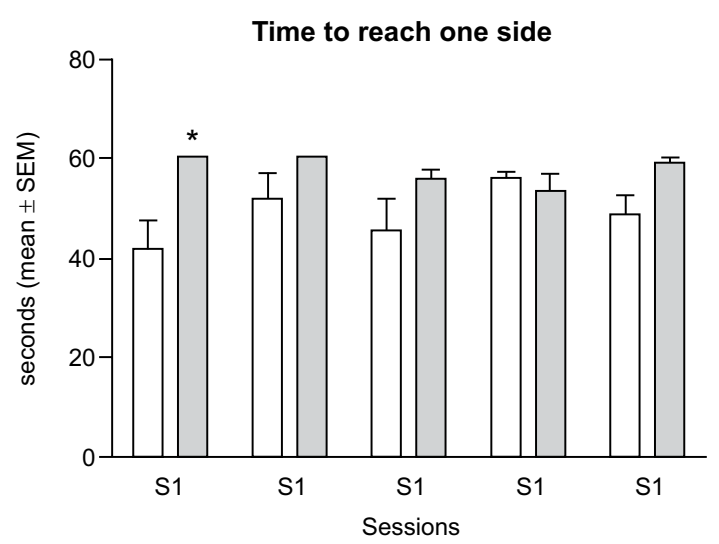

Figure 2. Effects of prenatal LPS exposure (100 $\mu \mathrm{g} / \mathrm{kg}$ on GD 18) on (A) the number of pups that chose the odor side, (B) the time (in seconds) to reach the odor side in five trials, and $(\mathrm{C})$ the time (in seconds) to reach the nest bedding area in the maternal odor preference test in infant male rat pups on PND 7. $n=8$ pups/group. The number of pups that chose the odor, neutral, and clean sides and time required to reach the nest were analyzed by two-way ANOVA followed by the Bonferroni test. The time to reach the odor side was analyzed by Student $t$-test. * $p<.05$, compared with the control group. Data are expressed as mean $\pm \mathrm{SEM}$. 

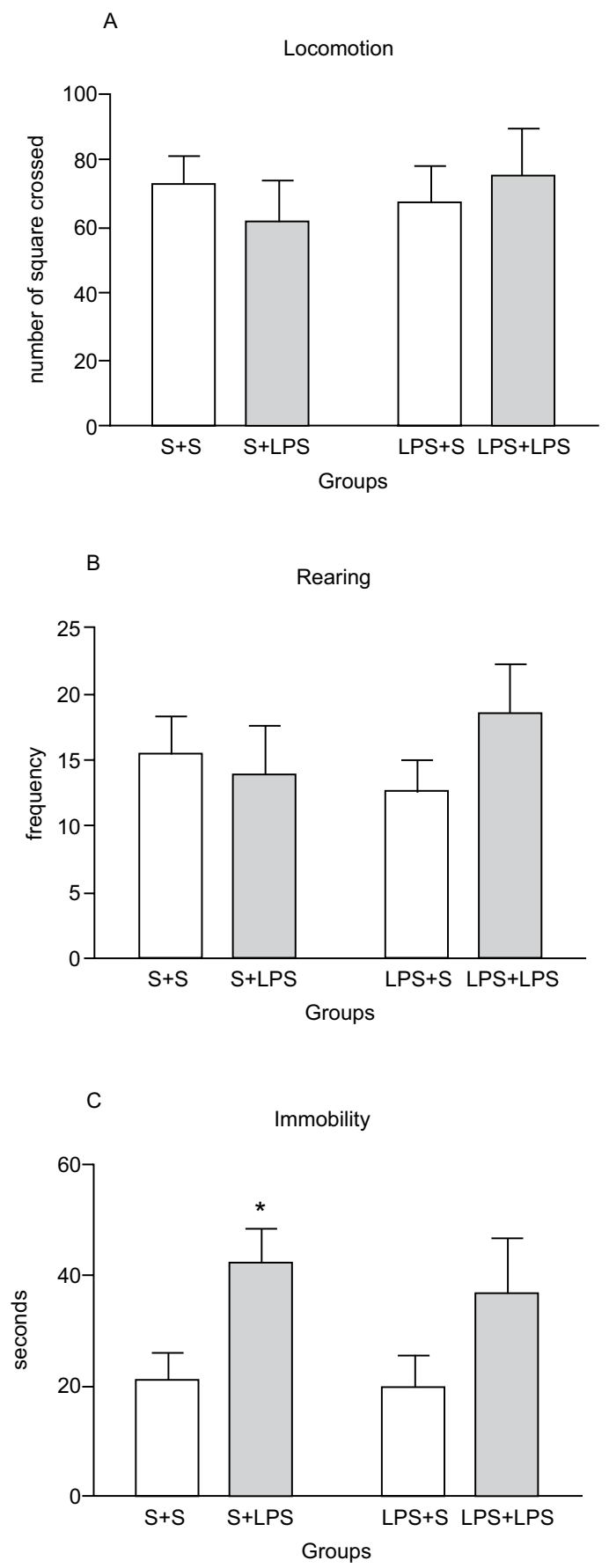

Figure 3. Open field behavior observed on LD 21 in male pup rats prenatally exposed to LPS on GD 18 and challenged with $50 \mu \mathrm{g} / \mathrm{kg}$ LPS. (A) Locomotor frequency. (B) Rearing frequency. (C) Immobility duration (in seconds). $n=8$ /group. ${ }^{*} p<.05$, compared with control group (two-way ANOVA followed by Bonferroni test). Data are expressed as mean \pm SEM.

$p=.75)$. The Bonferroni test revealed that immobility duration increased in the S+LPS group compared with the other groups.

Figure 4 shows TNF- $\alpha$ levels determined by ELISA. The two-way ANOVA revealed significant effects of prenatal treatment $\left(F_{1,27}=10.12, p=.0038\right)$ and postnatal treatment $\left(F_{1,27}=11.05, p=.0026\right)$, with a significant interaction between factors $\left(F_{1,27}=9.98, p=.0039\right)$. The Bonferroni test revealed significant differences

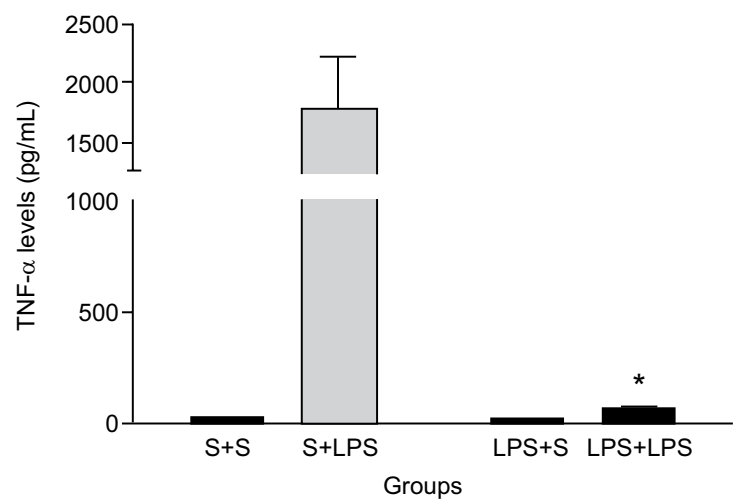

Figure 4. Serum TNF- $\alpha$ levels $(\mathrm{pg} / \mathrm{ml})$ on LD 21 in male pups prenatally exposed to LPS on GD 18 and challenged with $50 \mu \mathrm{g} / \mathrm{kg}$ LPS. *p $<.05$, compared with control group (two-way ANOVA followed by Bonferroni test). Data are expressed as mean \pm SEM.

between the LPS+S and LPS+LPS groups $(p<.001)$ but not between prenatal treatments (i.e., between the $\mathrm{S}+\mathrm{S}$ and LPS + S groups; $p>.05$ ). Thus, the S+LPS group exhibited an increase in TNF- $\alpha$ levels compared with the $\mathrm{S}+\mathrm{S}$ group. However, the LPS+LPS group exhibited a weak increase in TNF- $\alpha$ levels compared with the S+LPS group, indicating decreased sensitivity to LPS when the pups were prenatally exposed to the endotoxin. Additionally, only pups exposed to prenatal LPS and not exposed to postnatal LPS (i.e., the LPS + S group) exhibited detectable serum TNF- $\alpha$ levels.

\section{Discussion}

The present findings showed that prenatal LPS exposure (100 $\mu \mathrm{g} / \mathrm{kg}$ on GD 18) improved maternal care and reduced maternal aggressive behavior but did not alter maternal reproductive performance. With regard to male offspring, prenatal LPS treatment increased body weights at birth and reduced the preference for maternal odor. On LD 21, a challenge dose of LPS increased the duration of immobility in the open field test. This challenge dose also induced tolerance reflected by a slight increase in serum TNF- $\alpha$ levels in pups in the LPS+LPS group compared with the LPS+S group.

Maternal care improved in females prenatally exposed to LPS. These females exhibited a reduced latency to retrieve the first pup compared with the control group. With regard to maternal aggressive behavior, these females had fewer numbers of attacks and a reduced duration of time spent fighting than females in the control group. These apparently contradictory data may be explained by the mechanism of action of LPS.

Several authors reported that LPS affects central nervous system activity, leading to sickness behavior in many species (Aderem \& Ulevitch, 2000; Avitsur et al., 1997; Saluk-Juszczak \& Wachowicz, 2005). The innate immune system is responsible for many of the acute symptoms of sickness that are related to systemic inflammation or infection (Medzhitov \& Janeway, 1999; Rivest, 2003). Lipopolysaccharide-induced sickness behavior is generally accompanied by decreases in 
exploratory activity, social behavior, ingestive behavior, and sexual behavior. It also induces anhedonia and poor learning and cognitive function (Corrreia et al., 2008). Among the signs of LPS-induced sickness behavior, fever has also been reported (Hart, 1988). Fever, an adaptive reaction to pathogens (Mackowiak, 1998), results from a complex reaction in hypothalamic centers that inform the organism that the environmental temperature is low and induce an increase in body temperature (Voltarelli \& Loughran Junior, 1994). At birth, rodent neonates have not yet developed thermoregulatory mechanisms, and maternal care is critical to maintain pup survival. Both pup retrieval and nest building are important for the thermoregulation of pups. Retrieving behavior is directly linked to the survival of the litter and plays a major role in increasing the dam's inclusive fitness, defined by the number of offspring that survive and reproduce (Aubert, Goodall, Dantzer, \& Gheusi, 1997)

Recent studies from our group found that 100 $\mu \mathrm{g} / \mathrm{kg}$ LPS administered on LD 3 induced fever and increased maternal behavior (Nascimento, Felício, \& Bernardi, 2011) with a peak 48 and $72 \mathrm{~h}$ after endotoxin administration. No fever was observed $120 \mathrm{~h}$ after treatment. In the present study, maternal behavior was observed $192 \mathrm{~h}$ after LPS administration. Thus, maternal fever during pregnancy may signal to the mother that the environmental temperature is too low, and this sensory information may specifically increase the motivation of the dam to retrieve her pups.

Data from the maternal aggressive behavior test are apparently contradictory when compared with data from the maternal behavior test. However, the reduced number of attacks and reduced fight duration can be interpreted as an effect of LPS on motivation (i.e., a central state that organizes perception and action (Spencer, Martin, Mouihate, \& Pittman, 2006). We suggest that LPS may induce a competing motivational state characterized by reduced attention toward external events or an increased sensitivity threshold to external cues such as pup vocalizations, leading to increased preference to care for the pups and decreased aggressive responses toward intruders. Direct behavioral observations of the intruders indicated no aggressive behavior directed toward the pups.

In the present study, LPS treatment on GD 18 impaired the preference of male pups for maternal odor. We studied maternal odor preference only in male offspring because maternal behavior is much higher toward male pups than female pups (Francis, Champagne, \& Meaney, 2000). Therefore, we evaluated only male pups because any deficit in maternal behavior may be more apparent. Transient motor effects of prenatal LPS treatment were observed only in the first session in which the time to reach one side decreased in experimental pups compared with the control group. Therefore, the impaired olfactory preference was likely unrelated to impaired motor behavior. This hypothesis is supported by data collected at weaning when locomotor behavior in the prenatal LPS group (LPS+S group) did not differ from the control group.
In addition to olfaction impairment, another explanation for the present findings is that rats prenatally treated with LPS had impaired motivation. Support for this hypothesis comes from previous studies in which prenatal LPS exposure impaired social interest in rats during infancy and adulthood because of motivational impairments (Kirsten, Taricano, Maiorka, PalermoNeto, \& Bernardi, 2010a). Maternal olfactory stimuli are linked to rats' survival. The hypothesis that the observed effects reflect a reduction of motivation is intriguing but requires further investigation.

To test the sensitivity of pups to LPS-induced sickness behavior, we administered an additional dose of LPS and observed exploratory behavior in the open field. Prenatally saline-treated pups challenged with LPS at weaning exhibited an increased duration of immobility compared with the other groups. No changes were observed in the other open field parameters compared with the other groups. This effect on immobility may be correlated with the development of sickness behavior. However, prenatally LPS-treated pups that were also treated with LPS at weaning (LPS+LPS group) exhibited no changes in immobility, suggesting lower sensitivity to LPS-induced sickness behavior. Thus, activation of the immune system at the end of pregnancy may decrease the sensitivity to LPS-induced sickness behavior in prepubertal rats. To verify this hypothesis, we measured serum TNF- $\alpha$ levels. Surprisingly, pups that were prenatally treated with LPS did not exhibit LPS-induced immune system activation at weaning. Thus, prenatal exposure to LPS may induce immunological tolerance to LPS early in life. Lipopolysaccharide exposure during late pregnancy may negatively regulate LPS signaling and cause the development of tolerance. Antiinflammatory cytokines might play important roles in such a process. In vitro tolerance in human monocytes can be partially mimicked by interleukin-10 (IL-10) and TGF- $\beta$, and the use of anti-IL-10 and anti TGF- $\beta$ antibodies during the development of tolerance can prevent endotoxin tolerance (Randow et al., 1995).

In conclusion, prenatal exposure to LPS during late pregnancy improved maternal care but reduced maternal olfactory preference in male pups. Tolerance to a challenge dose of LPS was observed in pups prenatally exposed to the endotoxin.

\section{Acknowledgements}

This study was part of the doctoral thesis of Sandra Heloisa Nunes Whitaker Penteado and supported by CNPq (PIBIC), FAPESP grant 2010/01855-1, Thematic Award 09/51886-3, and UNIP grant 7-02-839/2012.

\section{References}

Aderem, A., \& Ulevitch, R. J. (2000). Toll-like receptors in the induction of the innate immune response. Nature, 406(6797), 782-787.

Aubert, A., Goodall, G., Dantzer, R., \& Gheusi, G. (1997). Differential effects of lipopolysaccharide on pup retrieving and nest building in lactating mice. Brain, Behavioral, and Immunity, 11(2), 107-118. 
Avitsur, R., Pollak, Y., \& Yirmiya, R. (1997). Different receptor mechanisms mediate the effects of endotoxin and interleukin-1 on female sexual behavior. Brain Research, 773(1-2), 149-161.

Bernardi, M. M., Kirsten, T. B., Matsuoka, S. M., Teodorov, E., Habr, S. F., Penteado, S. H., et al. (2010). Prenatal lipopolysaccharide exposure affects maternal behavior and male offspring sexual behavior in adulthood. Neuroimmunomodulation, 17(1), 47-55.

Boksa, P. (2010). Effects of prenatal infection on brain development and behavior: a review of findings from animal models. Brain, Behavior, and Immunity, 24(6), 881-897.

Broadhurst, P. L. (1960). Experiments in psychogenetics: Applications of biometrical genetics to the inheritance of behaviour. In H. J. Eysenck (Ed.), Experiments in personality (Vol. 1, pp. 1-256). London: Routledge \& Kegan Paul.

Burd, I., Balakrishnan, B., \& Kannan, S. (2012). Models of fetal brain injury, intrauterine inflammation, and preterm birth. American Journal of Reprodutive Immunology, 67(4), 287-294.

Carlos, C. P., Lemonica, I. P., de Grava Kempinas, W., \& Marques Pereira, O. C. (1996). Does the male reproductive performance depend on the early lactation milk in rats? Physiology and Behavior, 59(1), 147-152.

Corrreia, M. S. F., Fernandes, S. C., \& Bernardi, M. M. (2008). Effects of lipopolysaccharide (LPS) administration on stereotypy and catatonia of male adult mice. Journal of Health Science Institute, $26,215-220$

Darnaudery, M., \& Maccari, S. (2008). Epigenetic programming of the stress response in male and female rats by prenatal restraint stress. Brain Research Reviews, 57(2), 571-585.

de Moura, E. G., Lisboa, P. C., \& Passos, M. C. (2008). Neonatal programming of neuroimmunomodulation--role of adipocytokines and neuropeptides. Neuroimmunomodulation, 15(3), 176-188.

Francis, D. D., Champagne, F. C., \& Meaney, M. J. (2000). Variations in maternal behaviour are associated with differences in oxytocin receptor levels in the rat. Journal of Neuroendocrinology, 12(12), 1145-1148.

Golan, H. M., Lev, V., Hallak, M., Sorokin, Y., \& Huleihel, M. (2005). Specific neurodevelopmental damage in mice offspring following maternal inflammation during pregnancy. Neuropharmacology, 48(6), 903-917.

Graciarena, M., Depino, A. M., \& Pitossi, F. J. (2010). Prenatal inflammation impairs adult neurogenesis and memory related behavior through persistent hippocampal TGFbeta1 downregulation. Brain, Behavior, and Immunity, 24(8), 1301-1309.

Hart, B. L. (1988). Biological basis of the behavior of sick animals Neuroscience Biobehavioral Reviews, 12(2), 123-137.

Kirsten, T. B., Chaves, G. P., Taricano, M., Martins, D. O., Florio, J. C., Britto, L. R., et al. (2011). Prenatal LPS exposure reduces olfactory perception in neonatal and adult rats. Physiology and Behavior, 104(3), 417-422.

Kirsten, T. B., Taricano, M., Florio, J. C., Palermo-Neto, J., \& Bernardi, M. M. (2010b). Prenatal lipopolysaccharide reduces motor activity after an immune challenge in adult male offspring. Behavioural Brain Research, 211(1), 77-82.

Kirsten, T. B., Taricano, M., Maiorka, P. C., Palermo-Neto, J., \& Bernardi, M. M. (2010a). Prenatal lipopolysaccharide reduces social behavior in male offspring. Neuroimmunomodulation, 17(4), 240-251.

Kirsten, T. B., Taricano, M., Maiorka, P. C., Palermo-Neto, J., \& Bernardi, M. M. (2010b). Prenatal lipopolysaccharide reduces social behavior in male offspring. Neuroimmunomodulation, 17(4), 240-251.

Kirsten, T. B., Taricano, M., Maiorka, P. C., Palermo-Neto, J., \& Bernardi, M. M. (2010a). Prenatal lipopolysaccharide reduces social behavior in male offspring. Neuroimmunomodulation, 17(4), 240-251.

Mackowiak, P. A. (1998). Concepts of fever. Archives International Medicine, 158(17), 1870-1881.

Medzhitov, R., \& Janeway, C. A., Jr. (1999). Innate immune induction of the adaptive immune response. Cold Spring Harbor Symposia Quantitative Biology, 64, 429-435.

Meyer, U. (2011). Developmental neuroinflammation and schizophrenia. Progress in Neuro-Psychopharmacology \& Biological Psychiatry, 42, 20-26.

Meyer, U., Feldon, J., \& Fatemi, S. H. (2009). In-vivo rodent models for the experimental investigation of prenatal immune activation effects in neurodevelopmental brain disorders. Neuroscience \& Biobehavioral Reviews, 33(7), 1061-1079.

Meyer, U., Nyffeler, M., Engler, A., Urwyler, A., Schedlowski, M., Knuesel, I., et al. (2006). The time of prenatal immune challenge determines the specificity of inflammation-mediated brain and behavioral pathology. Journal of Neuroscience, 26(18), 47524762.

Nascimento, A. F., Felício, L. F., \& Bernardi, M. M. (2011). Role of neurochemistry in the adaptive value of behavior: Competition between maternal behavior and the sick during lactation. Paper presented at the XXVI Reunião Anual da Federação de Sociedades de Biologia Experimental (FeSBE), Rio de Janeiro.

Randow, F., Syrbe, U., Meisel, C., Krausch, D., Zuckermann, H., Platzer, C., et al. (1995). Mechanism of endotoxin desensitization: Involvement of interleukin 10 and transforming growth factor beta. Journal of Experimental Medicine, 181(5), 1887-1892.

Rivest, S. (2003). Molecular insights on the cerebral innate immune system. Brain, Behavior, and Immunity, 17(1), 13-19.

Saluk-Juszczak, J., \& Wachowicz, B. (2005). The proinflammatory activity of lipopolysaccharide. Postepy Biochemii, 51(3), 280-287.

Slotnick, B., \& Restrepo, D. (2005). Olfactometry with mice. Current Protocols in Neuroscience, Chapter 8, Unit 820.

Spencer SJ, Mouihate A, Galic MA, Ellis SL, \& Pittman Q. J. (2007). Neonatal immune challenge does not affect body weight regulation in rats. American Journal of Physiology-Regulatory, Integrative and Comparative Physiology, 293(R), 581-589.

Spencer, S. J., Martin, S., Mouihate, A., \& Pittman, Q. J. (2006). Earlylife immune challenge: defining a critical window for effects on adult responses to immune challenge. Neuropsychopharmacology, 31(9), 1910-1918.

Teodorov, E., Felício, L. F., \& Bernardi, M. M. (2010). Maternal behavior. In FAPESP (Ed.), Animal models as Ethical tools in Biomedical Research. (Vol. 1). São Paulo: Andersen.M.L.,Tufik, S

Voltarelli, J. C., \& Loughran Junior, T. P. (1994). Expansion of a subset of TCR gamma/delta human lymphocytes from various lymphoid organs cultured with recombinant IL-2. Brazilian Journal of Medical and Biological Research, 27(3), 709-718.

Wang, X., Rousset, C. I., Hagberg, H., \& Mallard, C. (2006). Lipopolysaccharide-induced inflammation and perinatal brain injury. Seminars in Fetal \& Neonatal Medicine, 11(5), 343-353.

Wilkins, A. S., Logan, M., \& Kehoe, P. (1997). Postnatal pup brain dopamine depletion inhibits maternal behavior. Pharmacology Biochemistry and Behavior, 58(4), 867-873. 